\title{
ABSTRACTION AS A NATURAL PROCESS OF MENTAL COMPRESSION
}

\author{
Eddie Gray \& David Tall \\ Mathematics Education Research Centre \\ University of Warwick, Coventry, CV4 7AL, UK \\ <david.tall@warwick.ac.uk; e.m.gray@warwick.ac.uk>
}

\section{INTRODUCTION}

The term 'abstract' has its origins in the Latin $a b$ (from) trahere (to drag) as:

- a verb: to abstract, (a process),

- an adjective: to be abstract, (a property),

- and a noun: an abstract, for instance, an image in painting (a concept).

The corresponding word 'abstraction' is dually a process of 'drawing from' a situation and also the concept (the abstraction) output by that process. It has a multimodal meaning as process, property or concept. Piaget distinguished between construction of meaning through empirical abstraction (focusing on objects and their properties) and pseudo-empirical abstraction (focusing on actions on objects and the properties of the actions). Later reflective abstraction occurs through mental actions on mental concepts in which the mental operations themselves become new objects of thought (Piaget, 1972, p. 70). In Tall et al, 2000, we reviewed ideas in the literature and concluded that elementary mathematical thinking uses reflective abstraction both by focusing on objects (for instance, in geometry) and on operations on objects represented as symbols (in arithmetic, algebra, etc). In the latter case we see symbols used dually as process and concept and have formulated this in terms of the notion of procept (Gray \& Tall, 1994, see also below). At a later stage, in advanced mathematical thinking, the focus changes to properties (of objects and operations) formulated as fundamental axioms for mathematical theories.

Our hypothesis is that different forms of abstraction lead to different type of cognitive development and in turn, to differing cognitive problems. Empirical and reflective abstraction in shape and space lead to a van Hiele type development that we see as the growing dominance of verbal description over visual perception, as language refines our imagery and leads to increasingly sophisticated forms of mathematical structure and proof. Pseudo-empirical and reflective abstraction in arithmetic, algebra and calculus naturally focus on our notion of procept. Increasing focus on properties and deduction lead to a property-based axiomatic theory where the process of proof leads to the concept of theorem which may then be used as steps in building up a systematic formal theory.

We have a great empathy for the notion of different modes of operation as proposed by Bruner (1966) and, more particularly, in the SOLO taxonomy of Biggs and Collis. (1982). For instance, it is possible to build a holistic embodied mode that relates to the enactive/iconic modes of Bruner or the sensori-motor/ikonic modes of Biggs and Collis, before gaining an insight in proceptual (concrete-symbolic) terms; 
or, at a later stage in advanced mathematical thinking, in formal-deductive terms. Tall (1999) considers the distinct forms of proof available in these various modes as the child develops cognitively into a mathematical expert. Tall (2002) reviews calculus in terms of an enactive-iconic approach manipulating graphs, symbolicproceptual representations (manipulating formulae) and formal proof (in analysis).

In this short paper we do not have space to attend to our full theoretical perspective. We focus only on the abstractive processes occurring in constructing procepts in arithmetic, algebra and symbolic calculus and how differing types of symbol (whole numbers, fractions, algebraic expressions, (infinite) decimals, limits) give rise to distinct problems of concept construction and re-construction.

\section{FIVE ASPECTS}

The research forum is designed to focus on five aspects, given in (a)-(e) below.

\section{a. What is the theory about?}

Our theory grows as a result of our quest to understand not only what students do in constructing symbolic mathematics, but how they do it. We believe that abstraction is a natural consequence of human brain function. At any given time human thinking occurs dynamically as a process, whereby items evoked in the focus of attention are manipulated mentally as concepts. It is the duality of symbols in arithmetic, algebra, etc as both process and concept that is the basis of our theory.

\section{b. What assumptions are being made?}

We assume that abstraction is a natural product of human mental activity, in which a complex parallel-processing organ solves the problem of complexity by focusing on essential structures that enable decisions to be made. Sometimes this process of abstraction is a conscious reflective act, but much of it does, and must, occur unconsciously to enable the brain to focus only on essential elements. There is physical evidence that over time routinising tasks uses less brain capacity:

As a task to be learned is practiced, its performance becomes more and more automatic; as this occurs, it fades from consciousness, the number of brain regions involved in the task becomes smaller.

(Edelman \& Tononi, 2000, p.51)

There is also a compression in the nature of the symbolism being used:

I should also mention one other property of a symbolic system - its compactibility - a property that permits condensations of the order $F=M A$ or $S=1 / 2 g t^{2}$, ...in each case the grammar being quite ordinary, though the semantic squeeze is quite enormous.

(Bruner, 1966, p. 12.)

We do not have the data to link mathematical activity in a one-one mapping to neurophysical phenomena, steps in this direction (eg DeHaene, 1997) are still in their early stages. However, the underlying biological basis of mathematical thinking in a brain ill-built for numerical computation and formal logic, is a vital underpinning for our own reflections on how mathematical thinking develops. 


\section{c. What does the theory claim? What terms are used and what do they mean?}

The notion of procept (as given in Gray \& Tall, 1994) is seminal in what follows.

An elementary procept is the amalgam of three components: a process which produces a mathematical object, and a symbol which is used to represent either process or object.

... A procept consists of a collection of elementary procepts which have the same object. (Gray \& Tall, 1994, page 121).

We follow Davis (1983, p. 257) in defining a procedure as an explicit step-by-step algorithm for implementing a process and see a spectrum of increasing power through the usage of procedure, process and procept. We do not agree with Sfard or Dubinsky that the development invariably proceeds in a sequence we describe as procedure-process-procept. In particular, as students become more sophisticated, they may sense an intuitive holistic grasp of the overall ideas in, say, an embodied mode before concerning themselves with the specific procedures that may be seen to occupy a particular role within a symbolic or formal mode of operation.

We do not have a theory that tells us how all individuals can be helped to move through all of these modes. (Indeed, no-one has such a theory at this moment in time.) Instead, in the growth of symbols, we find a bifurcation between those who concentrate more on the procedures associated with symbols, who have a greater cognitive strain to overcome, and those who develop a proceptual system switching flexibly between process and concept to construct a more powerful generative mental structure. This does not mean that students necessarily remain in a fixed part of the spectrum. However, we do have considerable evidence that there is a bifurcation in performance between those who remain entrenched in procedures and those who develop more flexible proceptual thinking, so that progress to greater sophistication is more difficult for some and easier for others.

\section{d. What are the aims of the theory and what are its applications?}

The initial aim of our theory of the proceptual growth of symbols is to try to explain why some students are so highly successful with symbols, whilst others are procedural at best and could, at worst, be overwhelmed by the complexity of mathematics. To move towards this overall goal we focus on the different ways that procepts arise in cognitive development. These include

(1) arithmetic procepts, $5+4,3 \times 4,1 / 2+2 / 3,1 \cdot 54 \div 2 \cdot 3$, all have built-in algorithms to obtain an answer. They are computational, both as processes and even as concepts. Fractional procepts behave differently because the focus moves from sharing procedures (eg divide into 4 equal parts and take 2) to equivalent fractions, which from our viewpoint are seen as processes that have the same effect (divide into 4 equal parts and take 2, has the same effect as divide into 6 equal parts and take 3 ).

(2) algebraic procepts, e.g. $2+3 x$, can only be evaluated if the value of $x$ is known and so involves only a potential process (of numerical substitution) and yet the algebraic expressions themselves represent manipulable concepts (manipulated using algebraic rules of eauivalence). 
(3) implicit procepts, such as the powers $x^{1 / 2}, x^{0}$ or $x^{-1}$, for which the original meaning of $x^{n}$ no longer applies but the properties need to be deduced using the power law $x^{m} \times x^{n}=x^{m+n}$ ( which also no longer has its original meaning!)

(4) limit procepts, $\lim _{x \rightarrow a} \frac{x^{3}-a^{3}}{x-a}$ or $\sum_{n=1}^{\infty} \frac{1}{n^{2}}$ etc, have potentially infinite processes 'getting close' to a limit value that may not be computable in a finite number of steps.

(5) calculus procepts, such as $\frac{d\left(x^{2} e^{x}\right)}{d x}$ or $\int_{0}^{\pi} \sin m x \cos n x d x$ focus again on finite operational algorithms of computation (the rules for differentiation and integration). This reveals that each of new form of procept has its own peculiar difficulties that makes abstraction qualitatively different in each case. We believe that knowledge of these specific difficulties is essential to help a wider spectrum of students to succeed in the longer-term process of successive abstractions.

\section{e. How has the theory been validated?}

Our data (summarized in Tall, Gray, et al, 2001) reveals both general themes and specific information on cases (1)-(5) above. The general themes illustrate diverging approaches from procedural to proceptual in a spectrum of students from elementary arithmetic (Gray \& Tall, 1994), through algebra (DeMarois, 1998; McGowen, 1998; Crowley, 1999), symbolic calculus (Ali, 1996), and on to formal mathematical theory (Pinto, 1998). In addition, qualitative differences in imagery emerge from different forms of abstraction (Pitta, 1998; Gray \& Pitta 1999), leading to differing levels of success in the longer term, depending on whether children continue to focus on real-world situations and imagery, or move on to a more flexible proceptual hierarchy (Gray et al, 1999). The data from the above-mentioned studies reveal how differing contexts pose significantly different kinds of cognitive problems in both the nature of the procepts concerned and the procedure-process-procept spectrum of student activity. We believe that these difficulties are best handled by the learner supported by a mentor who is aware not only of the mathematics but of the underlying cognitive structures.

This aspect of learning is complementary to the desire of Dreyfus et al (this forum) to theorize about a general strategy for encouraging abstraction in context. We suggest that it is a laudable aim to have a general theory of construction, but we observe that specifics often overwhelm the broad sweep of such a theory. From the learner's point of view, different obstacles occur in different contexts. The acquisition of mathematical knowledge from early years to undergraduate level involves a variety of reconstructions. Each new reconstruction refines that which was established earlier so that effective reconstructions contribute to the organic nature of growth in the embodied and proceptual modes of operation and on to a close harmony between wider aspects of concept image and concept definition in advanced mathematical thinking. Our central concern is not just how we can encourage students to make abstractions, but also to find why some students succeed so 
effortlessly and others can fail so badly at making the necessary reconstructions. Our empirical evidence provides an insight into a possible answer-inappropriate abstraction from mathematical activity.

\section{References}

Ali, M. b. (1996). Symbolic Manipulation Related to Certain Aspects Such as Interpretations of Graphs, PhD Thesis, University of Warwick.

Biggs, J. \& Collis, K. (1982). Evaluating the Quality of Learning: the SOLO Taxonomy. New York: Academic Press.

Bruner, J. S. (1966). Towards a Theory of Instruction. Cambridge: Harvard.

Crowley, L. R. F. (2000) Cognitive Structures in College Algebra. PhD Thesis, Warwick.

Davis, R. B. (1983). Complex Mathematical Cognition. In Herbert P. Ginsburg (Ed.) The Development of Mathematical Thinking, (pp. 254-290). Academic Press, New York.

Dehaene, S. (1997). The Number Sense. New York: Oxford University Press.

DeMarois, P. (1998). Aspects and Layers of the Function Concept, PhD Thesis, Warwick.

Edelman, G. M. \& Tononi, G. (2000). Consciousness: How Matter Becomes Imagination. New York: Basic Books.

Gray, E. M. \& Tall, D. O. (1994). Duality, Ambiguity and Flexibility: A Proceptual View of Simple Arithmetic, The Journal for Research in Mathematics Education, 26, 115-141.

Gray, E. \& Pitta, D. (1999). Images and their frames of reference: A Perspective on Cognitive Development in Elementary Arithmetic, Proceedings of XXIII International Conference for the Psychology of Mathematics Education, 3, 49-57. Haifa: Israel.

Gray, E., Pitta, D., Pinto, M, Tall D.O (1999), Knowledge Construction and diverging thinking in elementary and advanced mathematics, Educational Studies in Mathematics, 38 (1-3), 111-133.

McGowen, M. C. (1998). Cognitive Units, Concept Images \& Cognitive Collages, PhD Thesis, Warwick.

Piaget, J. (1972). The Principles of Genetic Epistemology. London: Routledge \& Kegan Paul.

Pinto, M. M. F. (1998). Students' Understanding of Mathematical Analysis, PhD Thesis, Warwick

Pitta, D. (1998). Beyond the Obvious: Mental Representations and Elementary Arithmetic, $\mathrm{PhD}$ Thesis, Warwick

Tall, D. O. (1999). The Cognitive Development of Proof: Is Mathematical Proof For All or For Some? In Z. Usiskin (Ed.), Developments in School Mathematics Education Around the World, vol, 4, 117-136. Reston, Virginia: NCTM.

Tall, D. O. (2000). Biological Brain, Mathematical Mind \& Computational Computers. In Wei-Chi Yang, Sung-Chi Chu, Jen-Chung Chuan (Eds), Proceedings of the Fifth Asian Technology Conference in Mathematics, Chiang Mai, Thailand (pp. 3-20). ATCM Inc, Blackwood VA.

Tall, D. O, Gray, E M., Ali, M. b., Crowley, L. R. F., DeMarois, P., McGowen, M. C., Pitta, D., Pinto, M. M. F., Thomas, M., \& Yusof, Y. b. (2001). Symbols and the Bifurcation between Procedural and Conceptual Thinking, Canadian Journal of Science, Mathematics and Technology Education 1, 81-104.

Tall, D. O., Thomas M. O. J, Davis, G., Gray, E. M., Simpson, A. P. (2000). What is the object of the encapsulation of a process? Journal of Mathematical Behavior, 18 (2), 1-19. 